\title{
A computer-aided design tool for biomedical OBT sensor tuning in cell-culture assays
}

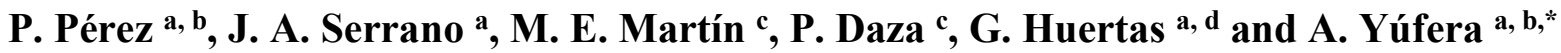 \\ ${ }^{a}$ Instituto de Microelectrónica de Sevilla (IMSE-CSIC), Av. Americo Vespuccio 24, 41092 Sevilla, Spain \\ ${ }^{\mathrm{b}}$ Departamento de Tecnología Electrónica, ETSII, Universidad de Sevilla, Av. Reina Mercedes sn, 41012, Sevilla, Spain \\ ' Departamento de Biología Celular, Facultad de Biología, Universidad de Sevilla, Av. Reina Mercedes sn, 41012, Sevilla, Spain \\ ${ }^{\mathrm{d}}$ Departamento de Electrónica y Electromagnetismo, Facultad de Física, Universidad de Sevilla, Av. Reina Mercedes sn, 41012, Sevilla, Spain
}

\begin{abstract}
:
Background and objectives: The biomedical engineering must frequently develop sensor designs by including information from performance of bio-samples (cell cultures or tissues), technical specifications of transducers, and constrains from electronic circuits. A computer program for realtime cell culture monitoring system design is developed; analyzing, modelling and integrating into the program design flow the electrodes, cell culture and test circuit's influences.
\end{abstract}

Methods: The computer tool, first, generates an equivalent electric circuit model for the cellelectrode bio-systems based on the area covered by cells, which also considers the cell culture dynamics. Second, proposes an Oscillation Based Test (OBT) parameterized circuit, for Electrical Cell-Substrate Sensing (ECIS) measurements of the cell culture system bioimpedance. Third, simulates electrically the full system to define the best system parameter values for the sensor.

Results: Reported experimental results are based on commercial gold electrodes and the AA8 cell line. Characteristics of the cell lines, as time-division or cell size, are incorporated into the program design flow, showing that for a given assay, the optimal OBT circuit parameters can be selected with the help of the computer tool. The electrical simulations of the full system demonstrate that the can be correctly predicted the output frequency and amplitude ranges of the voltage response, obtaining accurate results when cell culture approaches to confluence phase.

Conclusion: It is proposed a computer program for system design of biosensors applied to monitoring cell culture dynamics. The program allows obtaining confident system information by electrical stimulation. All system components (electrodes, cell culture and test circuits) are properly modelled. The employed procedure can be applied to any other $2 \mathrm{D}$ electrode layout or alternative circuit technique for ECIS test. Finally, deep insight information on cell size, number, and time-division can be extracted from the comparison with real cell culture assays in the future.

Submitted: 3 May 2020. Revised: 18 Sep 2020. Accepted: d mm yyyy

Keywords: Bioimpedance; cell culture; Computer Aided Design (CAD); microelectrode; Oscillation Based Test (OBT).

* Corresponding author 


\section{Introduction}

Biological matter observation is an important topic to better understand and diagnosis the living organisms existing on Earth, including ourselves. The analysis of the biological properties in a sample using non-invasive measurements is a powerful tool to visualize varied physical parameters, which can have deep biological and medical implications. The non-invasive approaches [1][2] can be applied to obtain information from a biological entity. The bioimpedance (BI) analysis stands out as a simple and easy technique that requires only the application of some electrodes in direct contact with the biological sample under test. This as leads to an easy interface between the sample to be measured and the circuits for test and analysis. Best reason underlying the selection of bioimpedance as biomedical marker is that all living matter has a direct dependence on its electrical properties given by the conductivity $(\sigma)$ and permittivity $(\varepsilon)$ parameters. These electrical properties can define the healthy or unhealthy status of any living cells, tissues, organs, full body etc., if their optimum or gold values dependence, in frequency and time, are known [3].

Fig. 1. Steps to design an impedance sensor to analyze biological samples from electrical measurements.

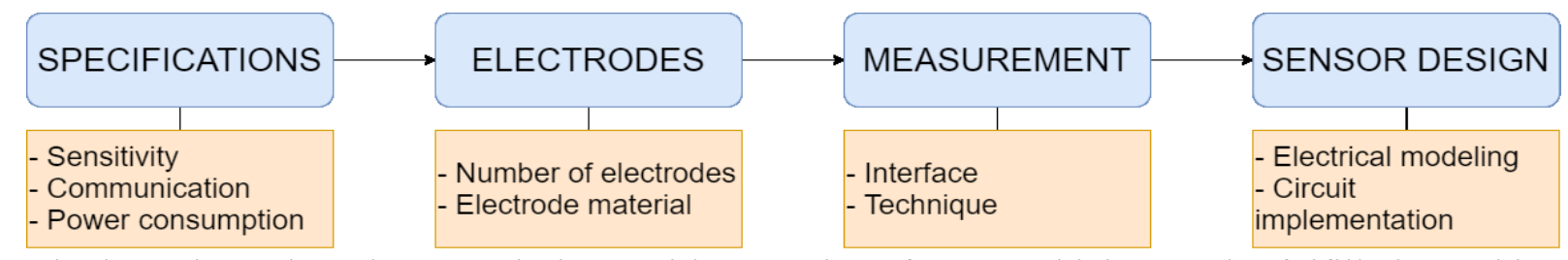

The impedance-based sensor design entitles a series of steps, which must be fulfilled to achieve adequate performance and desired outcomes. As starting point, the particular application should be defined. This one can reach many biomedical tests [4], with wide work conditions (clinical, labs, wearable or implanted devices, LoC, etc.). A first approach to solve the BI test measurement is the selection of the electrode setup. In general, four-electrodes setups are preferred due to the possibility to sense voltage signals non-destructively employing very high input-impedance voltage amplifiers [4]. However, the latest one is not always possible, and two-electrodes setups 
are mandatory [5]. Fig. 1 illustrates the general process, depicting the four main steps known: the specification elicitation the electrode selection the applied measurement technique, and finally, the sensor design and implementation. In the first phase, measurement specifications about test quality (expected magnitude and phase dynamic ranges, accuracy, frequency range, etc.) defined also the system features: power consumption, wireless communication, weight and size, etc. have to be detailed. To satisfy the input specifications, electrodes and test measurement circuits must be chosen. The electrode selection depends on material; size and number that enable the signal pickup without modify it as much as possible. Usually, gold ( $\mathrm{Au})$, platinum $(\mathrm{Pt})$ or stainless steel electrodes are employed because of their excellent biocompatibility and low resistance. A main drawback due to the requisite of using electrodes is the influence on test measurement derived from their electrical response. It means that the electrical parameters modelling the electrode performance: the Double Layer Elements ( $Z$ in Fig. $2 c)$ and the Spreading Resistance $\left(R_{s}\right.$ in Fig. $\left.2 c\right)$ will impact on test, particularly if the electrodes are located in the signal path. Therefore, electrode material and size must enable the correct measurement of the tested signal and to influence as less as possible on its measure. The selected BI technique [4], and the circuits to implement it should consider the general input specifications and the imposed constrains by the used electrodes. The most common approach employs a sinusoidal input current as excitation signal, and a look-in amplifier to extract the real and imaginary bioimpedance components from four-electrode setups [4][6][7]. The whole sensor design have to fulfil the input specifications, providing the BI values employing the best possible circuits designed for, bypassing the electrode influence introduced by its parasitic elements (capacitors and resistances).

In this paper, this design process will be tracked, and a Computer Aided Design (CAD) tool will be proposed following the above steps. Examples will be generated from our experience to illustrate every step: design, implementation and testing of biomedical sensors. A step-by-step 
guide will be provided showing the mandatory stages to obtain a successful biomedical design constrained [8] only by the applied technique. This work focus on the Oscillation Based Test (OBT) BI circuit technique [9][10].

The OBT is a technique derived from the microelectronic device testing field, which builds an oscillator as transducer for the electrical impedance associated to the sample under test. This approach, well documented on the literature for different applications [9]-[11], requires a solid knowledge of the sample under test prior to any sensing consideration. To test cell culture samples, where (cells are attached to the bottom substrate) this technique demands two electrodes interfacing the sample to the oscillation loop. The OBT system (bio-OBT when applied to biomedical sensing) then oscillates with an established frequency $\left(\mathrm{f}_{\text {osc }}\right)$ and amplitude $\left(\mathrm{a}_{\text {osc }}\right)$. Such oscillation parameters are mathematically modelled, and fluctuate depending on the sample under test, being different for cell-cultures, tissues or different biological samples.

The proposed design tool considers the evaluation of the cell culture growth by applying Electrode Cell Impedance Sensing (ECIS) approach [5]. The sensing process relies on the electro-chemical redox reaction occurring on an electrode immersed in an ionic solution (cell culture medium). The electrode creates an electrical path, modeled by analyzing the electro-chemical process [12][13]. This electrical path is modified by the adhesion of cells over the electrode surface. The Fig. 2 illustrates a basic physical schematic of the transduction process at the electrode (a), and the electrical models associated to different stages of the process (b) without cells on the sensing electrode, and (c) with a cell over the electrode.

The electrode model, the measurement analysis, and information recovered from the sensor output are required for the circuit design. Since there is also a certain amount of dispersion in electrode parameters, it will be necessarily considered it on the acquisition-processing algorithm. The electrode variations are inherent to the system, and there is no actual way to obviate it, hence a 
constructive approach would be to acknowledge its existence while analyzing the potential contribution to the resulting measurements and later information processing.

The cells growing over the electrode generate a secondary current path, which identifies the generation of a new passive $\mathrm{R}_{\text {gap }}$ resistance value. This path accounts for the contribution of the cell adhered to the electrode [14][15] and can change with the cell line. Others electrode models [19] consider that the parameters of interest (barrier resistance, cell radius and electrode-cell gap distance) are extracted only for the cell monolayer phase.

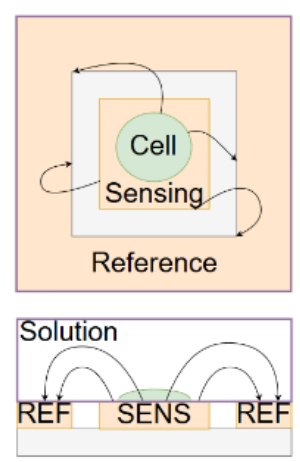

(a)

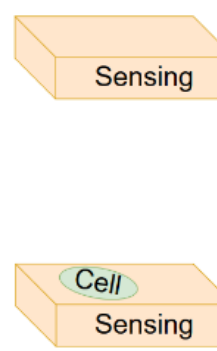

(b)
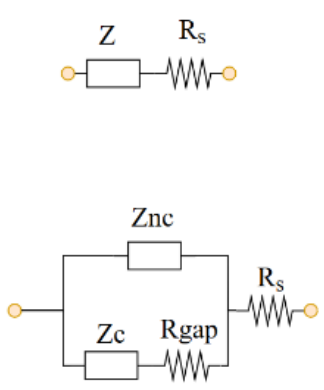

(c)

Fig. 2. Electrical models for cell culture over a sensing electrode. (a) TOP and SIDE views of the cell adhered to the electrode substrate immersed on an ionic solution. Two stages are depicted (and modeled); empty electrode (b) and partially covered electrode (c).

Sensitivity contributions of electrode parameters over the cell culture growth were analyzed and already reported in the literature [13][15]. They consider also the cell dynamic, which defines mainly the cell time-division value [18]. This paper will evaluate the design factors involved in the definition, design and implementation of an OBT based sensor for biomedical applications. 


\section{MATERIAL AND METHODS}

The structural components employed on the sensor design are firstly described in this section. Next, the cell culture dynamic incorporated on the design flow of the CAD tool is also considered. The image processing procedure employed for cell estimation is detailed, before to proceed to the electrical circuit simulation with Pspice as the final evaluation of the global sensor performance.

\subsection{Sensor design}

The physical characteristics to be measured on the sample under test are directly related with the impedance of the cell culture, and thus with the oscillation parameters delivered by the OBT based sensor. Its main building blocks are shown in Fig. 3. This simplified schematic incorporates the bioimpedance under test $\left(\mathrm{H}_{\mathrm{z}}(\mathrm{s})\right)$ using an inverter amplifier. Main blocks in the loop are the BandPass Filter $\left(\mathrm{H}_{\mathrm{BP}}(\mathrm{s})\right)$ that defines the operation frequency range for the oscillations, and the comparator. A detailed circuit description and analysis can be found in [10].

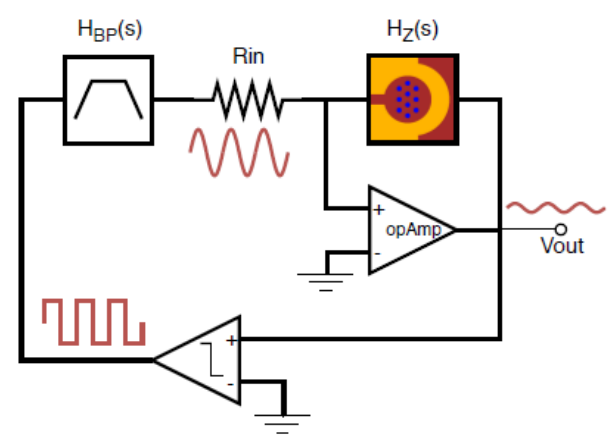

Fig. 3. Simplified schematic for the Oscillation Based Test (OBT) approach for bioimpedance measure. 

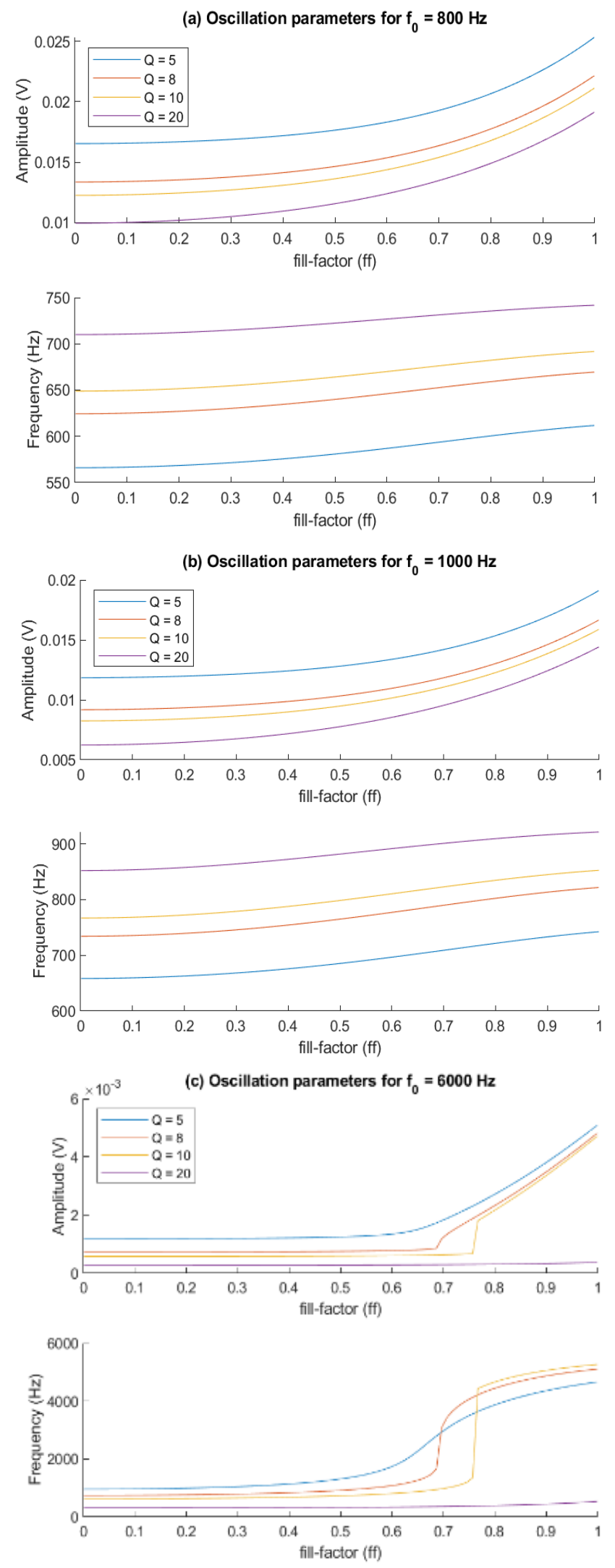

Fig. 4. Oscillation parameters; $\mathrm{f}_{\text {osc }}$ and $\mathrm{a}_{\mathrm{osc}}$, for several values for $\mathrm{Q}$ of the $\mathrm{BPF}$, and central frequencies of (a) 800 $\mathrm{Hz}$, (b) $1.000 \mathrm{~Hz}$ and (c) $8.000 \mathrm{~Hz}$. The curves consider from the electrode empty of cells ( $\mathrm{ff}=0)$, until the electrode is fully covered $(\mathrm{ff}=1)$. 
To produce an adequate electrical interface and define the filter parameters, a proper knowledge of the sample under test electrical model must be achieved. This model can be experimental or theoretical; however, it is necessary to perform an initial estimation, which will allow us as designers to find the optimal parameters. The employed model for the bioimpedance of the cell culture is described in Fig. 2, having as parameters, the double layer capacitance $\left(C_{d 1}\right)$; the transfer resistance $\left(R_{c t}\right)$ and the spreading resistance $\left(R_{s}\right)$ introduced by the electrodes in saline solution [12]; the parallel of $\mathrm{C}_{\mathrm{dl}}$ and $\mathrm{R}_{\mathrm{ct}}$ form $\mathrm{Z}$ in Fig. 2. Furthermore, the gap resistance $\left(\mathrm{R}_{\text {gap }}\right)$ derives from the cell influence, when cells are attached to the electrodes, covering part or all (confluence layer or monolayer) the electrode area. The fill-factor (ff) is a geometric parameter introduced to match the percent of the electrode area covered by cells (ff $\epsilon[0,1])$. The transfer function $\mathrm{H}_{\mathrm{z}}(\mathrm{s})$ describes the cell-electrode electrical performance, containing simultaneously the information about the frequency response and the area covered by the cells over the electrodes (ff). The ff value can be easily translated into cell density (cells $\left./ \mathrm{cm}^{2}\right)$ by knowing the electrode area and cell radius $\left(\mathrm{r}_{\text {cell }}\right)$ or area.

The OBT sensor requires the definition of a band pass filter (BPF) which will govern the sensor frequency and amplitude ranges affecting the sensor response [9][16]. Sensor curves are obtained from the electrical model of the impedance under test to evaluate for the optimal operation point in terms of sensitivity and performance while designing the system. The BPF parameters are: Q, quality factor; $\mathrm{f}_{\mathrm{o}}$, central frequency; and $\mathrm{G}$, gain of the passband. The sensor response obtained for $\mathrm{f}_{\text {osc }}$ and $\mathrm{a}_{\text {osc }}$ are shown in Fig. 4, using several central frequencies and quality factors. It was considered that electrodes are empty of cells $(\mathrm{ff}=0)$ and full $(\mathrm{ff}=1)$. The employed electrode electrical parameters are associated to the devices $8 \mathrm{~W} 10 \mathrm{E}$ from [13][17]. It can be appreciated how for $\mathrm{f}_{\mathrm{o}}=800 \mathrm{~Hz}$, Q values required are bigger than 5 . For $\mathrm{f}_{\mathrm{o}}=10 \mathrm{kHz}$, the frequency response is not 
monotonic. It is for $f_{o}=1 \mathrm{kHz}$ where we obtain the best dynamic range for $\mathrm{f}_{\text {osc }}$ and a asc with $\mathrm{Q}$ between 5 and 8 .

\subsection{Cell Growth Time-Evolution Model}

Beside the physical sensor model, a biological growth evolution analysis is necessary to simulate the dynamics of cells growing over the electrodes. Cell culture dynamics are extensively studied and is a process well documented in the literature [18]-[21]. On Fig. 5, the different stages for cell growth are illustrated. Cell culture experiences varying rates of growth depending on the current stage but for the purpose of developing a cell culture sensor, the third region identified in the illustration as exponential phase is our region of interest, given the practical coverage for the whole cell amount range. The cell number $\left(X_{t}\right)$ for this stage is characterized as:

$$
X_{t}=X_{t-1} \cdot e^{\mu(\Delta t)}
$$

Where $\mu$ is defined from the mean doubling time or generation time $(g)$ as:

$$
\mu=\frac{\ln 2}{g}
$$

The biological process simulation will be addressed on the third region (exponential phase), due to the fact that this region is containing most of the measurement range. The process involves image processing algorithms (derived from OpenCV library) and Pspice simulation capabilities. A general flowchart is presented on Fig. 6. First, at the initial time, cells are seeded on the electrodes selected (INITIAL_SEED). These cells will growth at the rate defined by their cell cycle by cell division (MITOSIS), landing again of the sensing electrode surface. The resulting fill factor from the mitosis process is evaluated with an imagen processing technique, identifying the electrode covered area (IMAGE_PROCESSING). Finally, the actual system is electrically simulated, considering the electrical model for the cell-electrode and the circuits proposed for measurement (PSIPICE_SIMULATION). As a result, there are obtained the values of amplitude and frequency 
for the OBT designed circuit that describes the actual cell culture status. This is repeated an integer number of times (i), below than the time window proposed ( $\mathrm{N}$ times the cell-line-cycle division period) for the experiment. The evolution of every step is detailed in the next paragraphs.

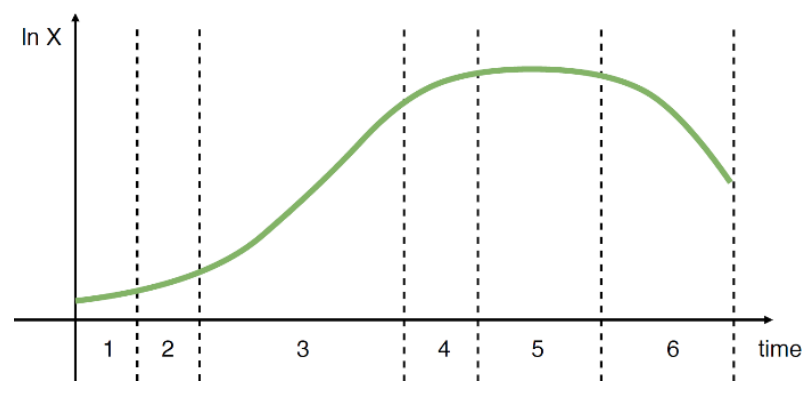

Fig. 5. Cell amount $(\mathrm{X})$ as a function of time. Adapted from [15]. Different growth phases for a cell culture. 1 (lag), 2 (acceleration), 3 (exponential), 4 (deceleration), 5 (stationary or confluent) and 6 (apoptosis).

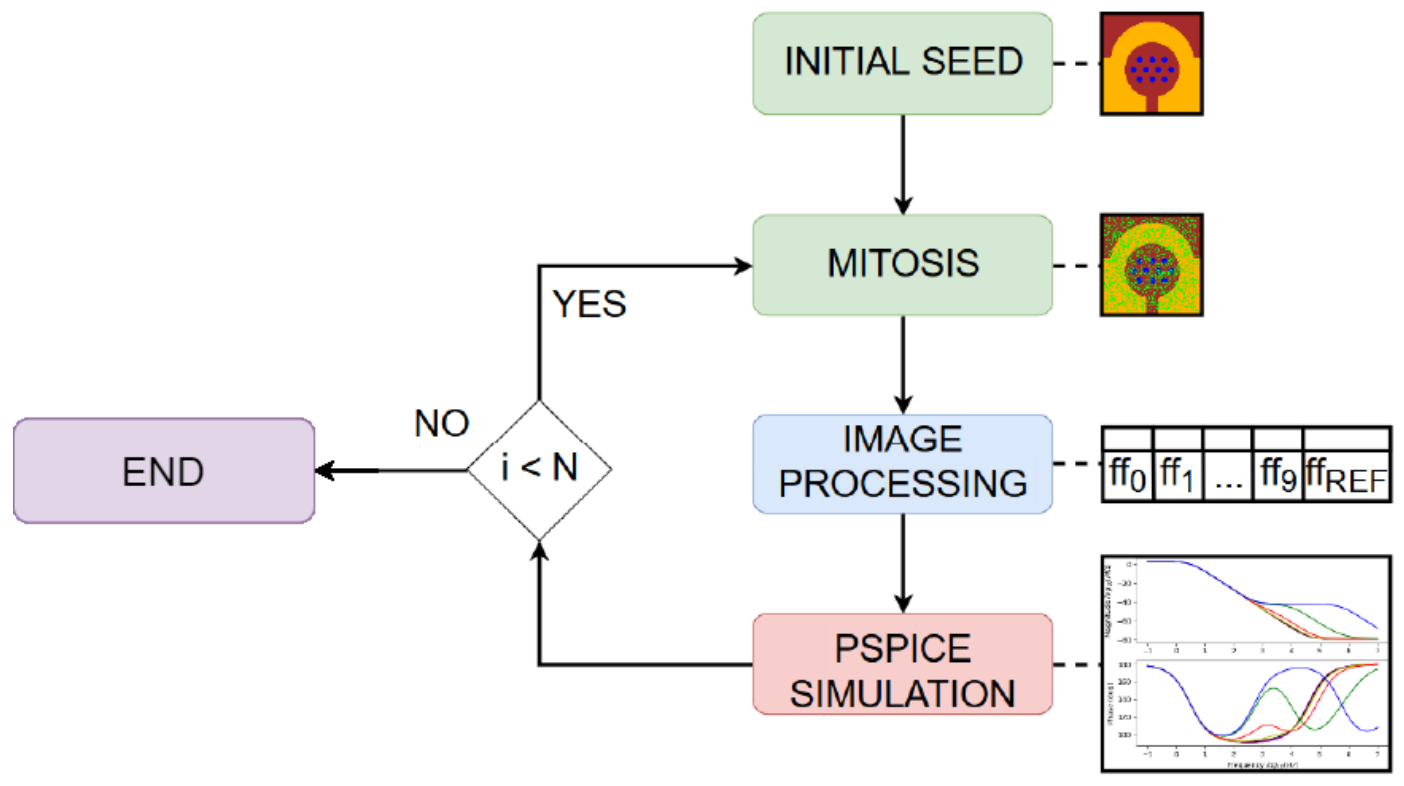

Fig. 6. Cell dynamics algorithm. The system requires several steps to analyze the electrical response of the cell dynamics modelled. 


\subsubsection{Initialization}

The algorithm first stage involves generating the raw images (modelled from the commercial electrode) and seeding the electrode with the initial number of cells. A circumference of radius, $\mathrm{r}_{\text {cell, }}$ composed by pure green pixels, models each cell (Fig. 7). The algorithm customizes initial position distributing the cells over the whole electrode area following an equidistant pattern, placing the cells randomly around the electrode or arranging them as a cluster, in any position. This is of great interest since not all electrodes will report similar behavior over a different cell arrangement due to electrode geometry.

\subsubsection{Mitosis}

The second stage emulates the natural division process known as mitosis occurring over the substrate in any cell culture experiment. An approach to the biological process undergoing is illustrated on Fig. 7. The depicted cell cycle is described by (2) assuming $g=t_{3}-t_{0}$. Any cell on the substrate is supposed to be adhered to it while not performing cell division (a). Before the mitosis happens, the cell will detach from the substrate (b) and initiate the biological process, which will eventually lead to a duplication (c). Finally, both cells will land again (d), adhering to the substrate at a certain distance $\mathrm{d}$ from the original position (e). This distance is modeled as a random distribution function around the original position. The mitosis stage updates the image on every iteration, creating the new cells and re-drawing the image upon cell landing computation. 


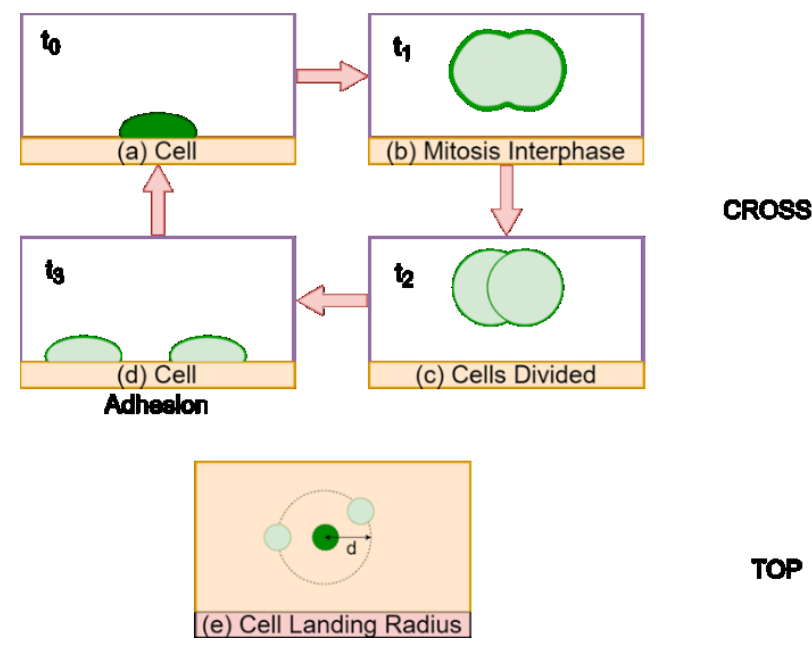

Fig. 7. Cell division cycle. A cell is adhered to the electrode surface on (a). To perform the mitosis, the cell detaches and start to divide (b), (c). After the division was performed, both cells land on the electrode surface (d) at a certain distance from the initial point (e).

\subsubsection{Image processing}

The third stage of the process involves the definition of the image processing algorithms, which will obtain the fill-factor value from the cell culture simulated images. Previously, the biological process was modelled as an image, which is evolving accordingly to the current cell growing time simulation. Analyzing a snapshot of the image at any moment can provide information on the number of cells over the electrode in simulation. This process can be extended to any electrode layout.

Each of the electrodes is modelled as a mask, which will be used to compute the area covered by the green pixels (cells) over each of the electrodes. This result is indeed the fill-factor (ff) value for each of the single electrodes implemented on the substrate, including the reference electrode (bigger one) in Fig. 8. 


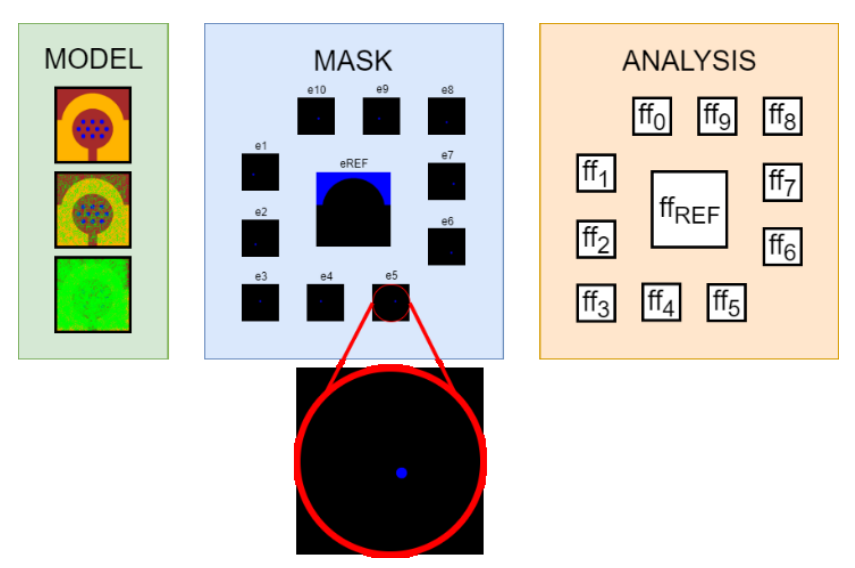

Fig. 8. Image processing algorithm to analyze the biological system and obtaining fill-factor (ff) values from the image generated using the modelled electrode and masks.

\subsubsection{Electrical Simulation}

The final stage requires the fill-factor (ff) values computed on previous state, along with the definition of the cell culture electrical model illustrated in Fig. 2. The associated ff value for each electrode allows the update of its electric model. From this, it is possible the definition of the corresponding Pspice netlist to perform the electrical simulation. Finally, the resulting information, frequency and amplitude of the signal oscillations, is extracted for each time value, allowing to assign a given value to each biological simulation. It is in this way how the expected sensor curve for the proposed sensing system is obtained and consequently, validated.

\section{Results and Discussion}

The system parameters were identified in terms of; the electrode model (derived from the electrical double layer) [13], the fill-factor (ff) [13], and the quality factor (Q), the central frequency $\left(\mathrm{f}_{\mathrm{o}}\right)$ and the filter gain $(\mathrm{G})$ defined in the OBT band pass filter [16]. The defined CAD tool integrates all those parameters, analyzing their impact for ECIS sensor design, as the main objective in this work. In parallel, a cell culture assay was performed to compare with the estimated results from the proposed $\mathrm{CAD}$ tool. In the experimental work were employed culturewares from $\mathrm{AB}$ [17] $8 \mathrm{~W} 10 \mathrm{E}+$. Each device has eight wells for separated cell cultures and every well with ten circular 
$\mathrm{Au}$ electrodes of $250 \mu \mathrm{m}$ diameter in parallel, forming the sensing electrode in Fig. 2a. The reference electrode for each well is much higher (top side in electrode layout Fig. 9), having a low impedance that can be neglected. The cell culture done for experimental comparison was carried out on a Chinese hamster ovary fibroblast cell line, AA8 (American Type Culture Collection). AA8 cells were cultured in McCoy's medium supplemented with 10\% (v/v) foetal calf serum, 2 mM Lglutamine, $50 \mu \mathrm{g} / \mathrm{ml}$ streptomycin, and $50 \mathrm{U} / \mathrm{ml}$ penicillin. Cells were routinely sub-cultured. The cell line was maintained at $37^{\circ} \mathrm{C}$ in a humidified atmosphere with $5 \% \mathrm{CO}$. They were always in exponential growth phase during the experiments.

Fig. 9 presents electrode selection tab, which allows the designer to select among different commercially available electrodes. The Electrical interface can be defined from the literature [12], being related with the electrode materials and the ionic solution. Typical values are presented as default, corresponding to cell culture medium and gold electrodes. Once the initial electrode configuration is selected, Fig. 10 corresponds to the second tab where the parameter $\mathrm{R}_{\text {gap }}[14]$ is selected and shall be defined. A typical value of $750 \Omega$ is considered by default; however, this value is could be highly dependent on the cell line under test [15].

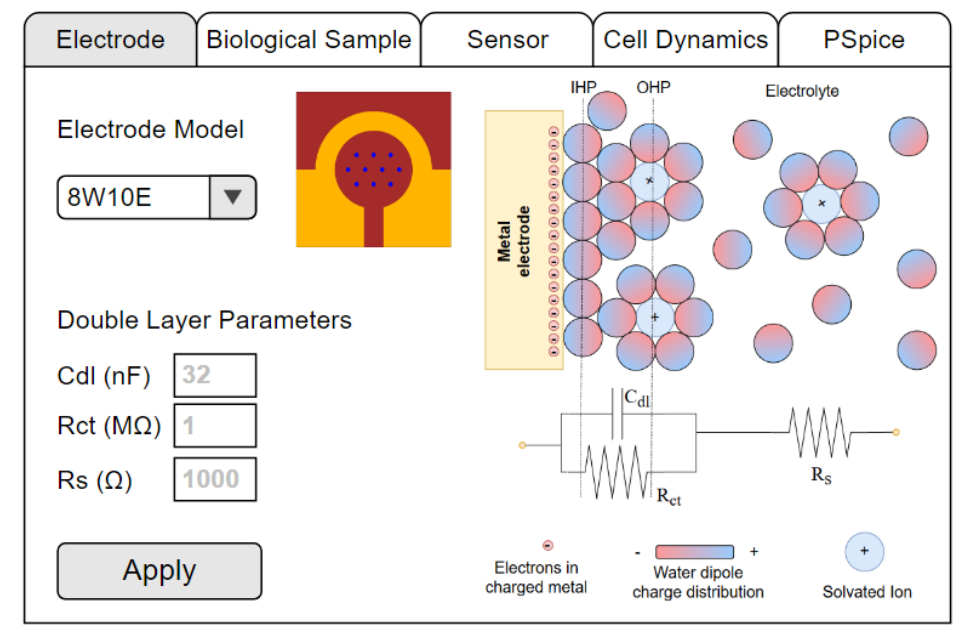

Fig. 9. Electrode settings tab for the ECIS CAD tool under development. 
The third tab (Fig. 11) configures the sensor parameter, which will define the sensor response provided herein, in terms of circuit response. The Q-factor, the central frequency and the filter gain (G) contribute to the output of the Oscillation Based Test sensor electrical response. Analyzing the contribution of such parameters along with the electrode and biological sample under test is crucial for an optimal sensor design.

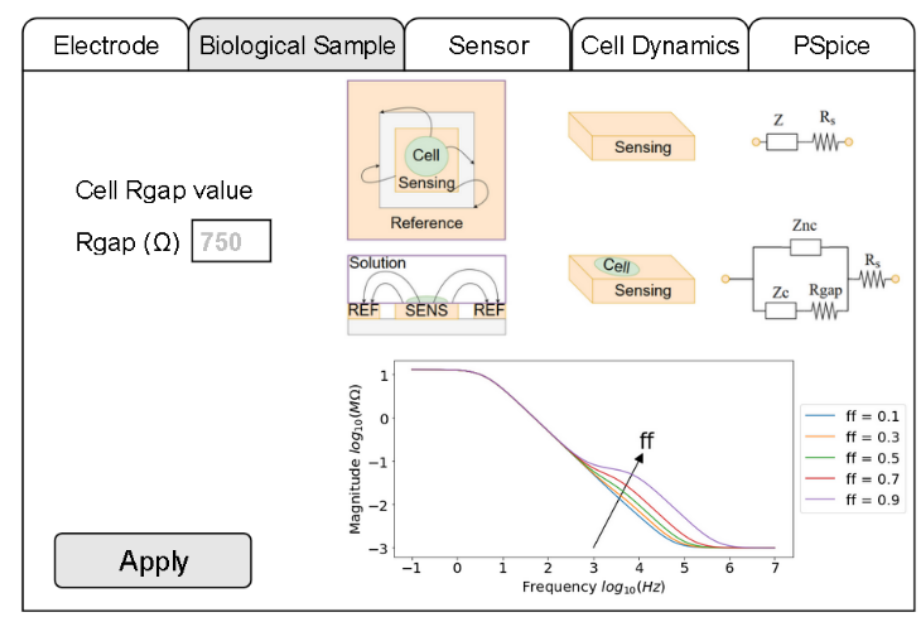

Fig. 10. Biological sample tab for the ECIS CAD tool under development.

The fourth tab (Fig. 12) establishes the biological simulation parameters (described on section II.B) which will model the cell growth over the electrode. Input parameters are the cell radius and time division (g parameter in eq. (2)); initial cell number and simulation step in hours, which is the time between snapshots. Hitting next will generate the snapshots and move the interface to the final tab entitled "Pspice", where the results of the electrical simulation are displayed for the OBT sensor. In particular, Fig. 13 displays the output results obtained seeding 16 cells in $\mathrm{t}=0$, with $\mathrm{r}_{\text {cell }}=25 \mu \mathrm{m}$ and a time division of 24 hours. The waveforms calculated for the sensor give a $f_{\text {osc }}=960 \mathrm{~Hz}$, and an $\mathrm{a}_{\mathrm{osc}}=10 \mathrm{mV}$ when reach an $\mathrm{ff}=0.9$ value. The observation time can be selected elsewhere. 


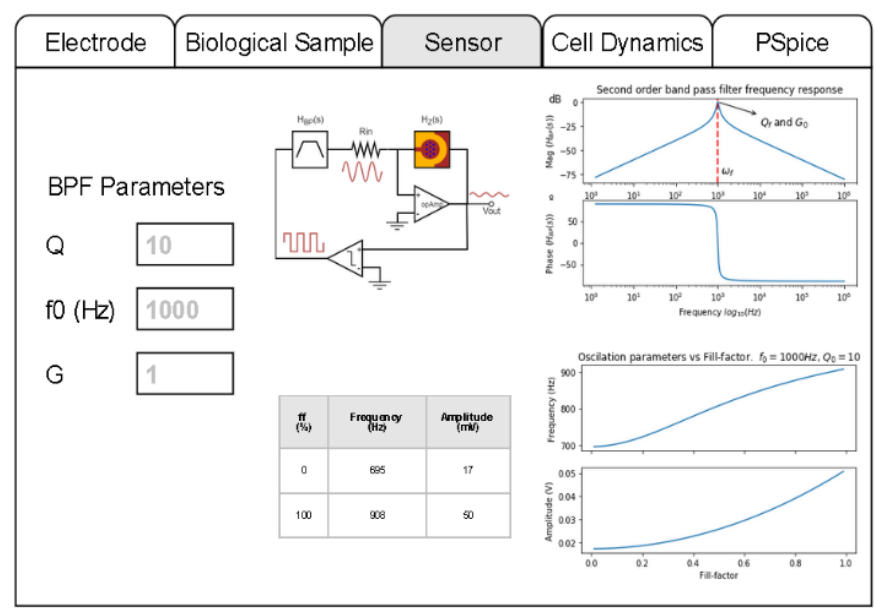

Fig. 11. Sensor tab for the ECIS CAD tool under development.

\begin{tabular}{|c|c|c|c|c|c|}
\hline Electrode & \multicolumn{2}{|c|}{ Biological Sample } & Sensor & Cell Dynamics & PSpice \\
\hline \multicolumn{6}{|c|}{ Dynamics Model } \\
\hline Random & $\nabla$ & & & & \\
\hline \multicolumn{6}{|c|}{ Cell Growth Parameters } \\
\hline \multirow{2}{*}{\multicolumn{2}{|c|}{$\begin{array}{l}\text { Cell Radius }(\mu \mathrm{m}) \\
\text { Initial Cell Number }\end{array}$}} & 25 & & & \\
\hline & & 16 & & & \\
\hline \multicolumn{2}{|c|}{ Time step (hours) } & 24 & & & \\
\hline Apply & & & & & Next \\
\hline
\end{tabular}

Fig. 12. Cell Dynamics tab for the ECIS CAD tool under development.

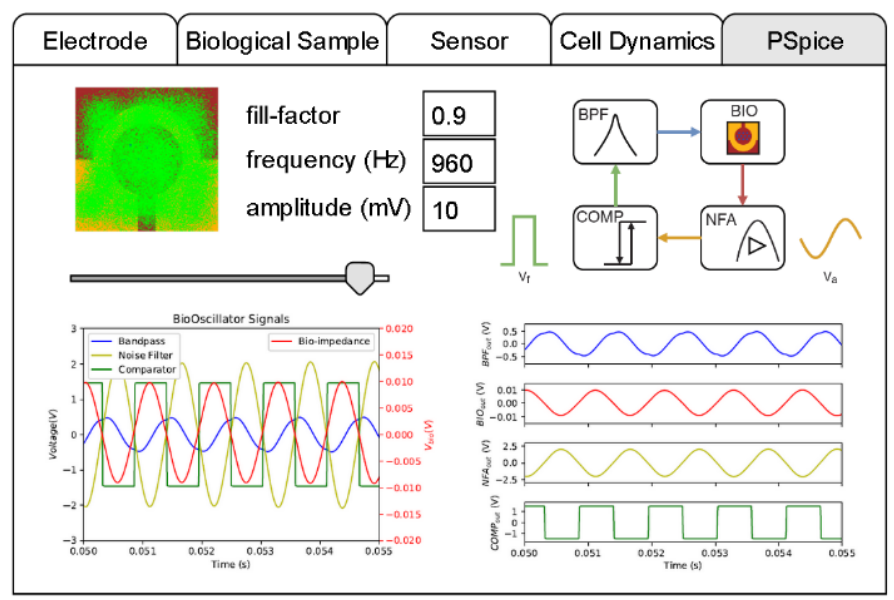

Fig. 13. Pspice tab for the ECIS CAD tool under development. 
To evaluate the accuracy of the tool, it was tested against real cell culture assay described before. The considered experimental data were reported in [10], employing the AA8 cell line. The AA8 cell line has a mean radius of $25 \mu \mathrm{m}$, and a time division of 24 hours. For the specific set of electrodes employed in the paper, the CAD tool reports a set of frequency and amplitude measurements expected from the Oscillation Based Test sensor. Using such equation set, the theoretical ranges for the amplitude and frequency values were predicted.

The experiment was performed seeding 2500 cells in a cell culture well from Applied Biophysics $8 \mathrm{~W} 10 \mathrm{E}+$. The results from such experiments are depicted on Fig. 14, together with the calculated by the presented tool.
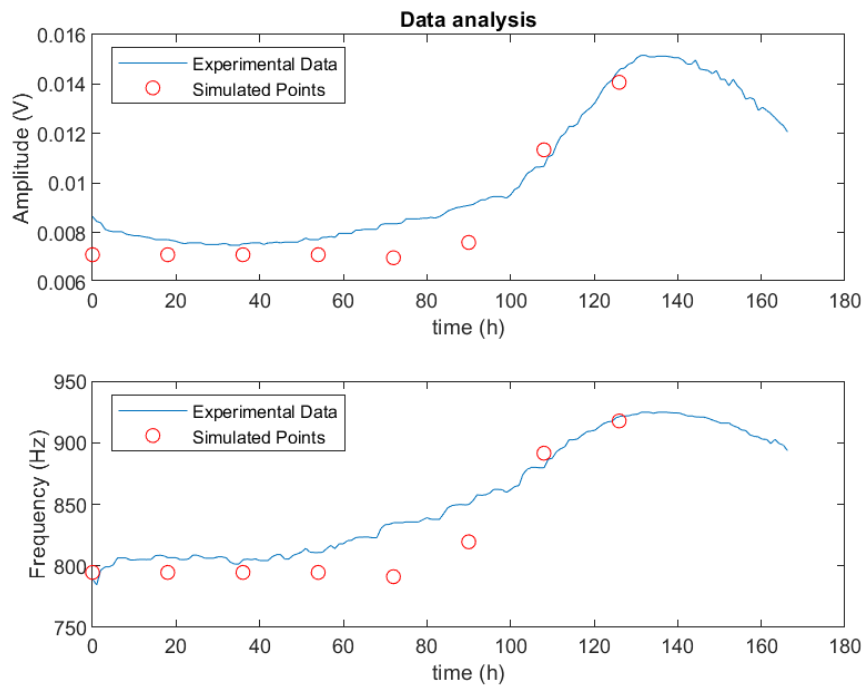

Fig. 14. Experimental results vs simulated points computed using the growth simulation.

Figure 14 presents both experimental and simulated results. First data corresponds to the continuous line obtained from sampling the cell culture (real laboratory experiment with a cell culture) every hour with the actual OBT circuit and computing the frequency and amplitude values from the sensor signal. Simulated information is presented as red points which illustrate the values obtained from the CAD tool execution. The modelled cell growth is computed assuming a cell 
radius of $25 \mu \mathrm{m}$, and a time division of $24 \mathrm{~h}$. It was employed the growth model evolution in time described before in section II.B. Both graphs depict a similar monotone increasing behavior and ranges.

Given the computational resources required to simulate huge amounts of cells dividing and randomly attaching to the surface of the electrode, an alternative approach was followed, considering a cluster of cells as the atomic element in the simulation. The cluster contains 325 cells seeded over a single circular gold electrode of $250 \mu \mathrm{m}$ diameter, and performs the division and attachment as reported previously. The red points are the simulated amplitude and frequency obtained from the electrical sensor model using the growth model as an input. The information contained in that comparison graph is relatively limited due to the characteristics of the growth model itself: it is employed only the exponential phase, and the limitations outlined in the paragraph above about the number of cells simulated. Probably, the our simulations will fit better to real performance if during the growing phase, illustrated in Fig. 5, we include also the lag (1), acceleration (2) and deceleration (4) phases, together with the exponential (3) one employed; applying then a more realistic growth model for the cell line. This requires a detailed programing of cell growth in time. A big limitation for to obtain a confident system simulation is the large number of cells in a culture. This can reach a density of cent of thousand cells by square centimeter, making unfeasible the description of all single cells. By increasing the computer power, this drawback can be partially reduced. Finally, some simulations parameters are homogeneous for all cells considered: size and time division for example. However, the cells can have different radius, even in the case they have a circular shape; question that it is not true. The elliptic shape could be a more realistic approach for cell shape. The same inhomogeneous performance can be applied to the constant time division of $24 \mathrm{~h}$ employed on simulations. 
An interesting value to consider here is the relation between the simulated fill-factor and the experimental fill-factor, which is presented in Fig. 15. The fill-factor obtained from the growth simulation is also accounting for the electrode shape and geometry, since the resulting value is obtained by computing the mean fill-factor value over each of the electrodes using the image processing techniques exposed previously. Computation of biological experimental results accounts for the fill-factor of the whole well (Area_well $=0.64 \mu \mathrm{m}^{2}$ ), considering the cell radius $\left(\mathrm{r}_{\mathrm{cell}}=25 \mu \mathrm{m}\right)$. An improved value could be obtained evaluating completely the well area $\left(0,64 \mathrm{~cm}^{2}\right)$, but this implies, as it is said before, a huge computational power to model the full cell system, and also, to simulate it.

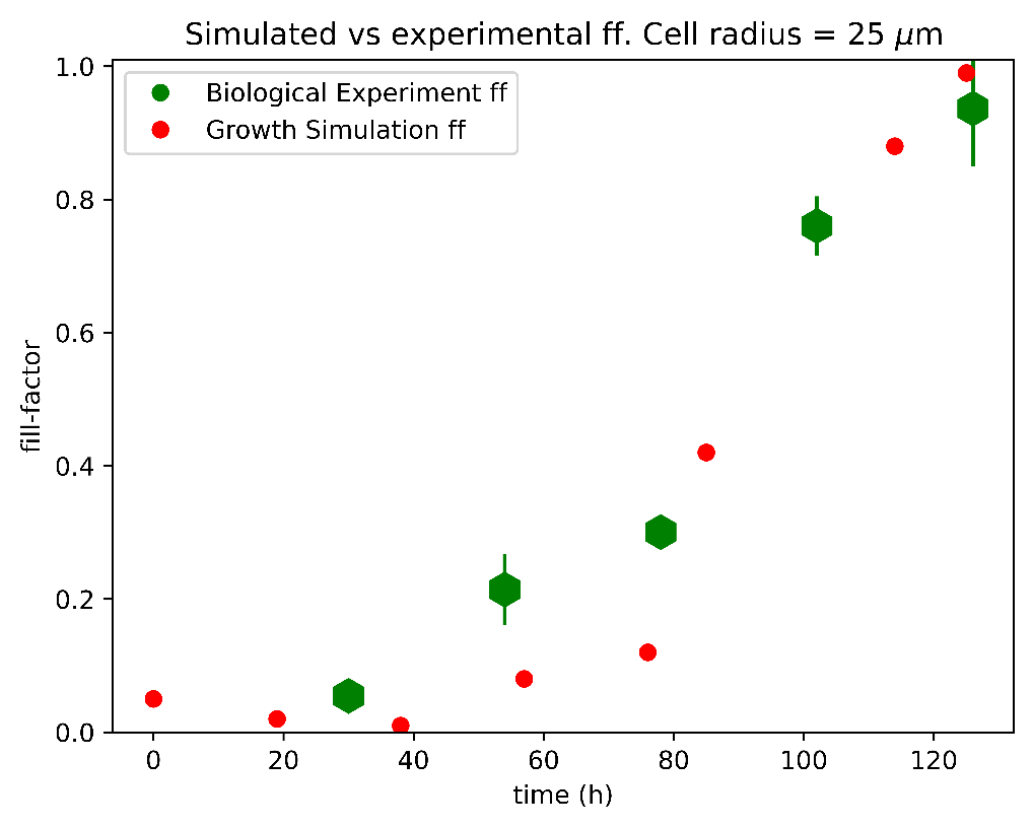

Fig. 15. Fill-factor versus time. Experimental results vs simulated points computed using the growth simulation.

\section{Conclusion}

The authors present a Computer Aided Design (CAD) approach for the assistance in Oscillation Based Test sensor designs by incorporating biological information from samples under test. The work presented here focus on the analysis of an ECIS based cell-culture sensor, carried out measuring the bioimpedance of cell-cultures with commercially available golden microelectrodes. 
The tool involves the technical information from circuits employed for BI test, with their design equations, the microelectrode electrical model, and the influence of cell-cultures on the observed bioimpedance, modelled with to the gap resistance parameter. Besides this static information, was modeled the dynamic evolution of cell-cultures since cells are living entities evolving in time; in particular, they are subjected to mitosis process periodically, as a function of its division time, increasing in time the number of cells in the culture. The proposed tool allows the update of the cell number in time to consider the real cell-culture status, and its influence on the circuit design. Image processing is applied to identify the cell number at each time instant. It is done by designing the identification masks associated to the electrodes employed but can be generalized to any electrode layout. As consequence, electrical circuit sensor and biology parameters are included simultaneously on the system, allowing achieving confident electrical simulations for validation of the proposed solution.

The tool was applied to sensor design in a practical case, and the experimental results obtained were appealing. It was confirmed that the predictions on frequency and amplitude oscillation ranges match the expected with the experimental ones. In addition, the time evolution prediction of the fill factor follows well the traditional cell density values measure optically, delivering a good method for cell culture confluent recognition. The errors in amplitude, frequency and fill-factor are lower when approach to confluent phase, being in general underestimated for low cell densities, mainly due to the limited surface covered by gold electrodes.

\section{Description of Competing Interest}

None

\section{Acknowledgements}

This work was supported in part by the Spanish Government's Ministerio de Ciencia, Innovación y Universidades, Plan Estatal 2017-2020 Retos- Proyectos I+D+I: Real time monitoring of 
hemodynamic variables using intelligent stents (iSTENT) with capacitive sensors and bioimpedance, under the project RTI2018-093512-B-C21, co-financed with FEDER.

\section{References}

[1] J. G. Webster, Medical Instrumentation Applications and Design, $4^{\text {th }}$ Ed. Wiley, 2009.

[2] Ping Wang and Qingjun Liu, Cell-Based Biosensors: Principles and Applications. Artech House, 2010.

[3] Schwan. H. P, Electrical properties of tissue and cell suspensions, Advances in Biological and Medical Physics, 5 (1957) 147-209.

[4] S. Grimnes and G. Martinsen, Bioimpedance and bioelectricity basics, Third ed., Academic Press, 2015.

[5] I. Giaever and C. R. Keese, Micromotion of mammalian cells measured electrically, Cell Biol, 88 (1991) 78-7900.

[6] M. Zamani, Y. Rezaeiyan, O. Shoaei, and W. A. Serdijn, A 1.55 $\mu$ W Bio-Impedance Measurement System for Implantable Cardiac Pacemakers in $0.18 \mu \mathrm{m}$ CMOS, IEEE Transactions on Biomedical Circuits and Systems, 12 (2018) 211-221.

[7] D. Allegri, A. Donida, P. Malcovati, and D. Barrettino, "CMOS-Based Multifrequency Impedance Analyzer for Biomedical Applications," in 2018 IEEE International Symposium on Circuits and Systems (ISCAS), pp. 1-5, IEEE, 52018.

[8] Hamid R Arabnia, Quoc-Nam Tran, Software tools and algorithms for biological systems, Springer. 2011.

[9] G. Huertas. Oscillation-based test in mixed-signal circuits. Springer. 2006.

[10] P. Pérez, G. Huertas, A. Maldonado-Jacobi, M. Martín; J. A. Serrano, A. Olmo, P. Daza, A. Yúfera, 2018. Sensing Cell-Culture Assays with Low-Cost Circuitry. Scientific Reports 8, 8841. 10.1038/s41598-01827295-3.

[11] A. Maldonado, G. Huertas, A. Rueda, J. L. Huertas, P. Pérez, A. Yúfera. Cell-culture measurements using voltage oscillations. IEEE 7th Latin American Symposium on Circuits \& Systems (LASCAS). (2016) 235238.

[12] D. Borkholder. Cell Based Biosensors Using Microelectrodes, Stanford University, 1998.

[13] P. Pérez. Circuit design for biomedical laboratories based on impedance measurement. PhD Thesis. Universidad de Sevilla, Jul. 2019.

[14] X. Huang; D. Nguyen; D. W. Greve; M. M. Domach. Simulation of Microelectrode Impedance Changes Due to Cell Growth. IEEE Sensors Journal, 4 (2004) 576-583.

[15] J. A. Serrano, G. Huertas, A. Maldonado-Jacobi, A. Olmo, P. Pérez, M. E. Martín, P. Daza, A. Yúfera, 2018. An Empirical-Mathematical Approach for Calibration and Fitting Cell-Electrode Electrical Models in Bioimpedance Tests. Sensors, 18, 2354. https://doi.org/10.3390/s18072354.

[16] G. Huertas, A. Maldonado, A. Yúfera, A. Rueda, J. L. Huertas, 2015. The Bio-Oscillator: A Circuit for CellCulture Assays. IEEE Transactions on Circuits and Systems II Express Briefs, 62 (2015) 164-168.

[17] AB ECIS Cell-based Assays from Applied BioPhysics. https://www.biophysics.com/

[18] B. O. Arredondo Vega, D. Voltolina, T. Zenteno Savín, M. Arce Montoya, G. A. Gómez Anduro, Métodos y Herramientas Analíticas en la Evaluación de la Biomasa Microalgal. 2017.

[19] I. Giaever and C. R. Keese, "Micromotion of mammalian cells measured electrically." Proceedings of the National Academy of Sciences of the United States of America, vol. 88, pp. 7896-900, 91991.

[20] J. R. Stein-Taylor, Handbook of phycological methods: culture methods and growth measurements; University Press, 1979.

[21] G. A. Harris, J. Coombs, D. D. Hall, S. P. Long, J. M. O. Scurlock, Techniques in Bioproductivity and Photosynthesis, 40, 2017. 